gekocht. Die dunkelblaue Lösung wird alkalisch gemacht und das unveränderte Dimethyl-m-toluidin mit Wasserdampf abgetrieben. Die hinterbleibende Farbbase wird in verdünnter Salzsäure gelöst und der Farbstoff mit Chlorzink gefällt. Er wird durch wiederholtes Lösen und Fällen gereinigt und färbt Wolle und Seide in reinen blauen Tönen an.

Zürich, Technisch-chemisches Laboratorium, Eidg. Technische Hochschule, 15. Juni 1918.

\title{
Sur la transformation de la lévoglucosane en dextrine
}

\author{
par \\ Amé Pietet.
}

(10. VII. 18.)

Dans une précédente communication ${ }^{1}$ ), nous avons montré, M. Jean Sarasin et moi, que l'amidon et la cellulose se convertissent tous deux en lévoglucosane, $\mathrm{C}_{6} \mathrm{H}_{10} \mathrm{O}_{5}$, lorsqu'on les distille sous pression réduite. Cette transformation pouvant être envisagée comme une simple dépolymérisation, s'effectuant selon l'équation

$$
\left(\mathrm{C}_{6} \mathrm{H}_{10} \mathrm{O}_{5}\right)_{\mathrm{n}}=\mathrm{n}_{6} \mathrm{H}_{10} \mathrm{O}_{5} \text {, }
$$

nous avions d'autre part $\left.{ }^{2}\right)$ manifesté l'intention de chercher à réaliser la réaction inverse, en soumettant la lévoglucosane à l'influence des agents polymérisants. M. Sarasin ayant quitté mon laboratoire, j'ai poursuivi seul l'exécution de ce programme. Elle s'est montrée plus facile que je n'osais l'espérer; j'ai trouvé, en effet, que la polymérisation de la lévoglucosane est aussi aisée que l'opération contraire, et qu'elle peut avoir lieu comme elle par la simple action de la chaleur. Il n'y a point, il est vrai, régénération de l'amidon ou de la cellulose, mais formation d'un

1) Helv. 1, 87 (1918).

2) C. K. 166, 38 (1918). 
terme intermédiaire, dont la grandeur moléculaire correspond à la formule $\left(\mathrm{C}_{6} \mathrm{H}_{10} \mathrm{O}_{5}\right)_{4}$ et qui, ainsi qu'on le verra plus loin, est une dextrine.

Lorsqu'on chauffe rapidement la lévoglucosane, elle fond, ainsi qu'il a été dit dans notre première note, à $180^{\circ}$; puis le liquide reste limpide jusqu'à $270^{\circ}$ environ, température à laquelle se produit une violente décomposition, avec formation d'eau, d'acides formique et acétique, d'acétone et de phẻnols. Un tout autre phénomène a lieu si l'on maintient la glucosane fondue à la température intermédiaire de $240^{\circ}$. Au bout d'un temps qui varie d'une demi-heure à une heure, on voit le liquide devenir visqueux et se boursoufler en se colorant en brun. Il n'y a ni dégagement gazeux ni changement de poids. La substance ne cristallise plus par refroidissement, mais se prend en une masse amorphe, dure et friable; celle-ci, à côté de lévoglucosane non transformée et de divers produits de décomposition, contient un nouveau corps, que l'on peut facilement isoler en dissolvant la masse dans l'eau froide et en ajoutant de l'alcool; il se dépose alors en flocons plus ou moins colorés, tandis que la lévoglucosane et les autres impuretés restent en solution. En répétant cette opération plusieurs fois, et en faisant bouillir les solutions aqueuses avec du charbon animal, on parvient à obtenir le nouveau composé à l'état parfaitement incolore.

Mais cette purification est longue et le rendement faible. La transformation est restée incomplète, ce qui doit être attribué sans doute au fait que la température de polymérisation de la lévoglucosane $\left(240^{\circ}\right)$ est trop rapprochée de sa température de décomposition $\left(270^{\circ}\right)$. Aussi ai-je cherché à l'abaisser par l'emploi de catalyseurs. Après quelques essais infructueux, j'ai trouvé dans le platine l'adjuvant dont j'avais besoin. Si, avant de chauffer la lévoglucosane, on la mélange avec une très faible quantité (un $100^{\mathrm{e}}$ ou même un $300^{\mathrm{e}}$ de son poids) de noir de platine, on voit la polymérisation commencer en même temps que la fusion de la substance. Il suffit de maintenir celle-ci à la température de $180^{\circ}$ pour que la transformation soit terminée en quelques minutes. On la voit débuter aux points où les parcelles de métal sont attachées aux parois du vase, et se propager de là dans toute la masse du liquide, qui se convertit en une matière solide, homogène et presque incolore. Celle-ci ne contient plus ni glu- 
cosane, ni produits de décomposition; après dissolution dans l'eau, elle en est intégralement reprécipitée par l'alcool, et le liquide filtré ne laisse par évaporation qu'un résidu insignifiant.

Je me hâte d'ajouter que les choses ne se passent d'une manière aussi nette qu'avec certains échantillons, particulièrement actifs, de noir de platine, et spécialement avec ceux que l'on a préparés suivant les indications de $L \propto w^{1}{ }^{1}$. Avec d'autres, la réaction est plus lente ou exige une température plus élevée; parfois même elle ne se produit pas. C'est là un fait qui a été souvent observé dans les catalyses par le platine, et dont on cherche encore l'explication.

Séché au bain-marie ou sur l'acide sulfurique, le produit de polymérisation de la lévoglucosane a l'aspect d'une poudre blanche et amorphe; retiré de ses solutions aqueuses par évaporation, il forme une masse transparente et vitreuse. Il n'attire pas l'humidité de l'air. Il se redissout lentement, mais abondamment dans l'eau froide; il est un peu soluble dans la pyridine, mais insoluble dans tous les autres dissolvants usuels.

Son analyse élémentaire a donné les résultats suivants:

I. 0,2082 gr. substance $-0,3398 \mathrm{gr} . \mathrm{CO}_{2}-0,1173 \mathrm{gr} . \mathrm{H}_{2} \mathrm{O}$

II. 0,2087 " $\quad 0,3403 ",-0,1155$,

\begin{tabular}{cccc}
\multicolumn{3}{c}{ Trouvé } & \multicolumn{1}{c}{ Calculé } \\
& I & II & pour $\mathrm{C}_{6} \mathrm{H}_{10} \mathrm{O}_{5}$ \\
C & 44,51 & $44,47 \%$ & $44,42 \%$ \\
H & 6,30 & $6,20 \%$ & $6,22 \%$
\end{tabular}

La détermination de son poids moléculaire a été faite par cryoscopie dans l'eau:

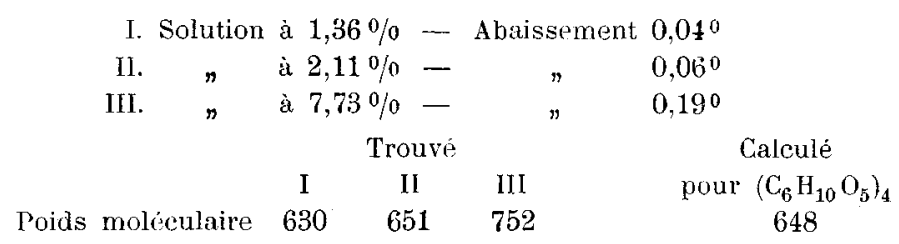

Etant donné qu'aux fortes concentrations on obtient toujours, par cryoscopie, des valeurs trop élevées du poids moléculaire, la dernière de ces trois déterminations me semble de nature à confirmer les premières, et à compenser ce qu'elles pourraient avoir d'un peu incertain du fait du faible abaissement observé.

1) B. $23,289(1890)$. 
J'estime done que l'on peut considérer la formule $\left(\mathrm{C}_{6} \mathrm{H}_{10} \mathrm{O}_{5}\right)_{4}$ comme représentant bien la grandeur moléculaire du produit de polymérisation.

Les solutions aqueuses du nouveau composé sont gommeuses, insipides et neutres au tournesol. Elles sont fortement dextrogyres; la polymérisation entraîne donc un changement de signe du pouvoir rotatoire. Quant à la valeur de celui-ci, les nombreuses déterminations que j'en ai faites ne m'ont pas conduit à des chiffres très constants. Il semble varier quelque peu avec la température à laquelle la substance a été portée, et être d'autant plus élevé que cette température est restée plus basse (racémisation?). Voici les résultats de deux de ces déterminations:

I. Substance préparée à $180^{\circ}$ :

$$
\varepsilon=1,555-1=1 \mathrm{dm}-a=+1,74^{\circ}
$$

II. Substance proparée à $200^{\circ}$ :

$$
\begin{gathered}
c=3,456-1=0,25 \mathrm{dm}-a=+0,920 \\
\text { I }
\end{gathered}
$$

Le produit de polymérisation n'est pas coloré par l'iode. Sa solution aqueuse n'est pas précipitée par le sulfate de soude, l'acide gallique, l'acétate de plomb ou l'eau de brome. Il ne réduit la solution de Fehling que faiblement et après une longue ébullition. Il ne fermente pas avec la levure de bière. Chauffé avec de l'acide sulfurique dilué, il se convertit en glucose (point de fusion de l'osazone $204^{\circ}$ ).

Ces propriétés sont celles qui caractérisent, d'une manière générale, les dextrines, et le nouveau composé doit indubitablement être rangé parmi les nombreux corps que l'on a réunis sous cette dénomination. Est-il identique à l'une des dextrines que l'on a obtenues par hydrolyse de l'amidon, du glycogène ou de la cellulose? Je ne saurais te dire actuellement. Il se distingue de la plupart d'entre elles par son poids moléculaire peu élevé et par son pouvoir rotatoire relativement faible. Il diffère, en outre, de l'amylodextrine et de l'érythrodextrine par le fait qu'il n'est pas coloré par l'iode; des maltodextrines par son pouvoir réducteur presque nul; des dextrines $\alpha$ et $\beta$ par sa plus grande solubilité dans l'eau et par l'absence de la saveur sucrée. Il se rapproche davantage de certaines achroodextrines, et en particulier de l'une de celles que l'on obtient dans l'hydrolyse de 
l'amidon par l'acide oxalique, et à laquelle $\left.W^{r} a c k e{ }^{1}\right)$ et von Friedrichs $\left.{ }^{2}\right)$ ont cru pouvoir assigner aussi la formule $\left(\mathrm{C}_{6} \mathrm{H}_{10} \mathrm{O}_{5}\right)_{4}$; toutefois le pouvoir rotatoire de cette dernière dextrine est notablement plus fort $\left(162,6^{\circ}\right)$.

Le corps que j'ai obtenu présente également certaines analogies avec les dextrines dites artificielles qui ont été préparées par Musculus et Meyer ${ }^{3}$ ) et par Grimaux et Le Fèvve ${ }^{4}$ ) en traitant le glucose par les acides sulfurique ou chlorhydrique, et qui possèdent un pouvoir rotatoire peu élevé $\left(97,5^{\circ}\right.$ et 131 à $\left.134^{\circ}\right)$. Mais ces deux auteurs s'accordent à attribuer à leurs produits une grande hygroscopicité et un poids moléculaire correspondant à la formule $\left(\mathrm{C}_{6} \mathrm{H}_{10} \mathrm{O}_{5}\right)_{3}$.

La question de l'identité de la nouvelle dextrine avec l'une ou l'autre de celles qui ont été décrites jusqu'ici ne saurait donc être résolue par mes observations. Ce qu'il me semble ressortir plus clairement de celles-ci, c'est la facilité inattendue avec laquelle la lévoglucosane, anhydride interne du dextrose, subit une première polymérisation, dès $180^{\circ}$, sous l'influence du noir de platine. J'ai l'intention de rechercher si, par la même voie de la catalyse, et à des températures supérieures, la polymérisation pourra être poussée plus loin. J'ai déjà pu constater que, lorsqu'on chauffe la dextrine à $250^{\circ}$ avec du noir de platine très actif, elle se convertit partiellement en une substance qui n'est plus soluble dans l'eau, mais qui se dissout un peu dans la liqueur de Schweizer.

Genève, Laboratoire de Chimie organique de l'Université, juillet 1918.

1) B. 42, $2675(1909)$.

2) C. $1914,1,761$.

3) Bl. [2] 35, 368 (1881).

4) C. R. 103, 146 (1886). 